\title{
IL-17A in Ovarian Cancer
}

\section{Takafumi Watanabe1, Masahiko Shibata ${ }^{2,3^{*}}$, Shu Soeda ${ }^{1}$, Shigenori Furukawa1, Keiya Fujimori', Koji Kono ${ }^{3}$, Seiichi Takenoshita ${ }^{1,2,3}$}

${ }^{1}$ Departments of Obstetrics and Gynecology, Fukushima Medical University, Fukushima City, Japan

${ }^{2}$ Comprehensive Cancer Treatments and Research at Aizu, Fukushima Medical University, Fukushima City, Japan

${ }^{3}$ Gastrointestinal Tract Surgery, Fukushima Medical University, Fukushima City, Japan

Email: ^mshibata@fmu.ac.jp

How to cite this paper: Watanabe, T., Shibata, M., Soeda, S., Furukawa, S., Fujimori, K., Kono, K. and Takenoshita, S. (2020) IL-17A in Ovarian Cancer. Journal of Cancer Therapy, 11, 605-616. https://doi.org/10.4236/jct.2020.1110051

Received: September 9, 2020

Accepted: October 11, 2020

Published: October 14, 2020

Copyright $\odot 2020$ by author(s) and Scientific Research Publishing Inc. This work is licensed under the Creative Commons Attribution International License (CC BY 4.0).

http://creativecommons.org/licenses/by/4.0/

\begin{abstract}
Ovarian cancer is the most common malignant disease leading to death among women. IL (interleukin)-17A is the most well-studied member of the IL-17 family, and has been demonstrated to play a critical role in host defenses against various microbial pathogens, as well as against tissue inflammation. T-helper (Th) 17 cells that produce interleukin (IL)-17A are of particular importance, because IL-17A exerts a wide variety of biological functions, particularly related to inflammation and the resultant carcinogenesis, as well as immune suppression in patients with cancer, and IL-17A-targeted therapy has been proven to be effective in the treatment of some autoimmune diseases. The pathogenic features of Th17 and IL-17A cells in cancer are still controversial, and Th17 cells appear to promote disease progression, as well as be present in the vicinity of many types of malignant diseases. In cancer patients, MDSC (myeloid-derived suppressor cells), one of the major immunosuppressive immature cells, and VEGF (vascular endothelial growth factor) are reported to correlate each other and strongly connected to IL-17-driven inflammation and malnutrition. In the present review, the latest advances are presented about the basic features of IL-17A and Th17. The function of IL-17A has not been clarified especially in ovarian cancer. This review overview the basic features of IL-17A and the functions in ovarian cancer as well as in other malignant and non-malignant diseases. Increasing our understanding of the interactions between IL-17A and ovarian cancer could lead to new therapeutic strategies in oncology.
\end{abstract}

\section{Keywords}

Ovarian Cancer, IL-17, VEGF, Immunosuppression, Disease Progression, Malnutrition 


\section{Introduction}

Ovarian cancer is the most common malignant disease leading to death among women [1]. The majority of patients are diagnosed at an advanced stage (III-IV) due to the late onset of symptoms, and the 5-year relative survival is only $29 \%$. Patients with advanced disease commonly develop an accumulation of ascitic fluid in the peritoneal cavity, facilitating the dissemination of tumor cells within the whole abdominal cavity. Standard treatment includes tumor debulking to surgically reduce the tumor load, followed by platinum-based chemotherapy. Despite improvements in the treatment of ovarian cancer, the majority of patients still relapse [2], and new treatment methods providing long-term efficacy are thus required.

The hallmarks of cancer represent a number of important characteristics required for the transformation of normal cells to malignant cells and tumor-promoting inflammation [3]. Inflammatory cells infiltrating the tumor microenvironment produce various growth factors and cytokines that sustain the proliferation of cancer cells, allowing them to resist cell death and promote invasion and metastasis [4]. Among these host-immune cells, T-helper (Th)17 cells that produce interleukin (IL)-17A are of particular importance, because IL-17A exerts a wide variety of biological functions, particularly related to inflammation and the resultant carcinogenesis, as well as immune suppression in patients with cancer [5]. IL-17A is the most well-studied member of the IL-17 family, and has been demonstrated to play a critical role in host defenses against various microbial pathogens, as well as against tissue inflammation [5].

While the role of IL-17A has been explored extensively in inflammatory diseases, its influence on the tumor-bearing host in malignant diseases remains to be fully defined. The function of IL-17A has not been studied, especially with regard to ovarian cancer. Here, we review the immunological mechanisms of IL-17 in cancer, and discuss the potential therapeutic strategy of IL-17 inhibition.

\section{IL-17A}

IL-17A, often referred to as IL-17, was originally discovered at the transcriptional level. Human and mouse IL-17A were later cloned, and IL-17A was demonstrated to be a major vehicle by which $\mathrm{T}$ cells communicate with the hematopoietic system and signal to bone marrow stromal cells to produce granulocyte colony-stimulating factor (G-CSF), which supports the differentiation of hematopoietic cells to the granulocyte lineage [6]. Subsequent studies have shown that IL-17A is produced in several types of cells. In 2005, Th17 cells were found to derive from naïve $\mathrm{CD} 4+\mathrm{T}$ cells under the control of TGF $\beta$ (transforming growth factor $\beta$ ) and IL-6, forming one of the major sources of IL-17A [7]. STAT3 and ROR $\gamma t$ were then demonstrated to play critical roles in Th17 lineage commitment in mice and humans [8] [9].

A receptor for IL-17A (IL-17RA) was first isolated and cloned in mouse thy- 
moma cells, and the bioactivity of IL-17A was confirmed in that same study [9]. IL-17RA is a member of the IL-17 receptor family, which includes IL-17RA, IL-17RB, IL-17RC, IL-17RD, and IL-17RE, and pairs with IL-17RC to allow binding and signaling of IL-17A and IL-17F [10] [11].

\section{Cellular Sources of IL-17A}

IL-17A is predominantly expressed by adaptive immune cell populations, including Th17 cells, cytotoxic T-cells, invariant natural killer T (NKT) cells, and $\gamma \delta \mathrm{T}$ cells [12].

Th17 cells belong to a subset of Th cells, characterized as producers of tumor necrosis factor (TNF) and IL-17, which represent the family of cytokines of IL-17A, IL-17B, IL-17C, IL-17D, IL-17E, and IL-17F, in addition to producing IL-6, IL-21, IL-22, IL-23 and IL-26 ([13], Figure 1). These are thus considered a new lineage of Th cells that play a key role in host defenses against many infectious agents by mediating effector mechanisms through their cytokines. The discovery of innate lymphoid cells (ILCs) has recently shown that IL-17A can also be produced by innate lymphoid cells and lymphoid tissue inducer-like cells [14]. Non-immune cells such as Paneth cells are able to secrete IL-17A [15]. Furthermore, Th17/IL-17A is also associated with chronic inflammatory diseases, autoimmune disorders, and cancer.

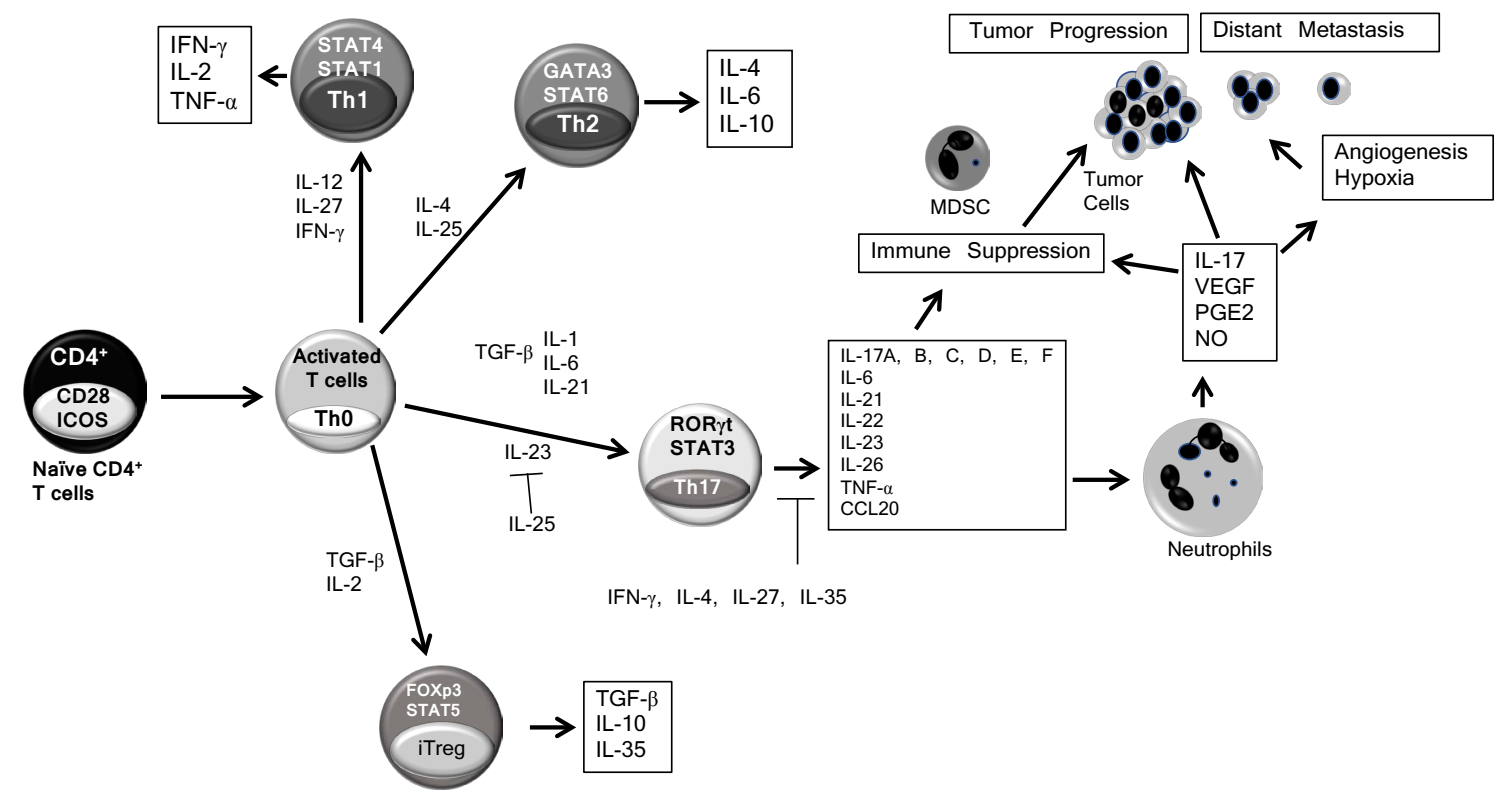

Figure 1. The lineage of Th17 and IL-17 promoting tumor progression. Th17 cells were found to derive from naïve CD4+ T cells under the control of TGF $\beta$ and IL-6, forming one of the major sources of IL-17A and other cytokines. STAT3 and ROR $\gamma t$ were then demonstrated to play critical roles in Th17 lineage. Several cytokines and chemokines including IL-17 and VEGF produced by the Th17 and neutrophils promote immune suppression involving MDSC, tumor progression and distant metastasis. IFN: interferon, IL: interleukin, TNF: tumor necrosis factor, Th: T-helper, TGF: transforming growth factor, iTreg: inducible regulatory T cells, CCL: CC chemokine ligand, PGE2: prostaglandin E2, NO: nitric oxide, MDSC: myeloid-derived suppressor cells, VEGF: vascular endothelial growth factor. 


\section{IL-17A in Host Defenses, Microbiota, and Carcinogenesis}

The primary function of IL-17A seems to be controlling gut microbiota as well as the clearance of extracellular bacteria and fungi [5]. The signaling of IL-17A coupled with IL-17 receptor has been shown to play a protective role in host defenses against many bacterial and fungal pathogens [16]. However, IL-17A does not appear protective against viral infections such as influenza [17].

Use of mouse models for colon cancer has provided evidence for interactions among innate and adaptive mucosal immune responses and colonic microbiota in colonic carcinogenesis. However, which members of the microbiota or which mucosal immune responses might promote colon cancer remains unclear [18]. Interestingly, IL-17A has been considered a strong candidate to promote inflammatory immunological responses leading to carcinogenesis [19]. The concept that microbe-induced IL-17A mucosal adaptive responses can be carcinogenic is supported by the observation that Helicobacter pylori-associated gastritis, which precedes most cases of gastric cancer, requires IL-17A [20]. However, studies to identify bacteria that induce IL-17A production in human gastrointestinal lesions are still ongoing. Mucosal Th17 cell-mediated immune responses have recently been suggested to require microbe-associated molecular patterns and physical proximity of the microbe to the epithelial cells [21].

\section{IL-17A in Autoimmune Diseases}

Th17 cells have been studied extensively in the past decade, and have been shown to be associated with the pathogenesis of many experimental autoimmune diseases and human inflammatory disorders. Continuing studies have suggested that IL-17A-producing cells, including Th17 cells, are involved in human psoriasis, rheumatoid arthritis, multiple sclerosis, inflammatory bowel disease, and asthma [22]. Although many studies have reported Th17 as the major source of IL-17A, non-lymphoid cells including mast cells and neutrophils may also produce IL-17A. This is because most of these conclusions have been based on immunohistochemical staining methods, which do not discriminate between IL-17A-producing cells and cells bound to IL-17A due to the ubiquitous expression of IL-17RA.

Multiple sclerosis is a neurological disease caused by immune cells that attack and destroy the myelin sheath insulating neurons in the brain and spinal cord. This neurological disorder and its animal model have historically been associated with the discovery of Th17 [23]. In addition, elevated IL-17A expression in disease lesions, as well as increased levels of IL-17A in peripheral blood, was reported prior to the discovery of Th17 [24].

Psoriasis, an autoimmune-inflammatory skin disease, is characterized by the infiltration of many types of immune cells, including massive infiltration of $\mathrm{CD} 4+$ cells, dendritic cells, cytotoxic $\mathrm{T}$ cells, and neutrophils in the dermis and epidermis [25]. Since a high production of interferon $\gamma$, TNF- $\alpha$, and IL-12 was present, psoriasis was recognized as a Th1-driven disease. However, since the 
discovery of IL-17A and Th17, Th17 cells have been suggested to synergize with Th1 cells in driving the pathologic pathway in the disease [26].

In rheumatoid arthritis, a chronic autoimmune-inflammatory disease with chronic joint inflammation that leads to destruction of cartilage and bone, IL-17A levels in the synovium have been reported to correlate significantly with tissue damage [27]. The direct clinical significance of IL-17A in rheumatoid arthritis was reported in recent clinical trial that found significant benefits from two anti-IL-17A antibodies (secukinumab and ixekizumab) [28].

Crohn's disease and ulcerative colitis, as two major types of inflammatory bowel disease (IBD) in human, have also been linked to IL-17A and Th17. According to Monteleone et al., massive Th-17 cell infiltration was evident in inflamed tissue in IBD and levels of IL-17A were elevated in patients with IBD [29]. However, recent clinical studies of agents targeting IL-17A have not shown any effectiveness, while increased adverse events were observed in the treatment arm [30].

\section{Systemic Inflammation and Ovarian Cancer}

Cancer-related inflammation is the hallmark of the tumor microenvironment, and has powerful effects on tumor development [3]. The tumor microenvironment in which ovarian cancer develops has been described as one enriched with a broad spectrum of proinflammatory cytokines and chemokines, which have been shown to influence clinical disease status and prognosis [31] [32]. Although IL-17A may promote the growth of ovarian cancer cells, little is known about the specific biological mechanisms through which IL-17A contributes to ovarian cancer initiation or progression. Recent studies have indicated that a direct linkage may exist between cancer stem-like cells (CSCs) and inflammation [33]. Xiang et al. suggested that IL-17A promoted self-renewal of ovarian CD133+ CSCs [34]. They also suggested that such stimulation of self-renewal in CD133+ CSCs might be mediated by NF- $\mathrm{kB}$ and the $\mathrm{p} 38$ mitogen-activated protein kinase signaling pathway, and summarized that the IL-17A-NFkB/p38 signaling network may be an ideal candidate for antitumor therapy against ovarian CSCs.

\section{IL-17A in Ovarian Cancer}

The pathogenic and non-pathogenic features of Th17 cells in cancer remain controversial. Th17 cells appear to promote disease progression in gastric cancer [35]. Th17 cells are also reportedly present in the vicinity of malignancies including ovarian, gastric, colorectal, breast, and pancreatic carcinomas and melanomas [36] [37] [38] [39] [40].

IL-17A can enhance tumor growth in vivo through the induction of IL-6, which in turn activates oncogenic transcription factor signal transducer and activator of transcription 3 (STAT3), and upregulates pro-angiogenic genes in tumors [41]. IL-17A also facilitates the development of colorectal carcinoma by fostering angiogenesis via promotion of vascular endothelial growth factor 
(VEGF) production by tumor cells [42]. However, the exact role of IL-17 in angiogenesis has yet to be determined (Figure 1).

Immunosuppressive myeloid cells have been described in cancer patients several times [43], although their functional significance in the immune system has only recently been evaluated. Accumulating evidence has suggested that a population of cells with suppressive activity, referred to as myeloid-derived suppressor cells (MDSCs), may contribute to the negative regulation of immune responses in patients with cancer [44] [45] [46] [47] [48]. We have previously characterized circulating numbers of MDSCs in patients with various types of cancers, and reported that increased production of IL-17A correlated with immune suppression involving MDSCs, as well as with malnutrition in patients with various types of cancer ([49] [50] [51] [52] [53], Figure 2).

As mentioned previously, many reports have described elevated IL-17A-producing cell counts and IL-17A expression in various cancers. Aotsuka et al. [54] demonstrated that occupancy ratios of Th17 and $\gamma \delta \mathrm{T} 17$ were increased in peripheral blood mononuclear cells (PBMCs) of patients with ovarian cancer. Moreover, neutrophil-to-lymphocyte ratio (NLR) correlated positively with the Th17 occupancy ratio and with both shorter overall survival and worse progression-free survival (PFS). They finally concluded that elevation of Th17/IL-17A, which is correlated with NLR/systemic inflammation or stimulation of PD-L1, offers a potential biomarker for predicting prognosis and PD-L1 overexpression in patients with ovarian cancer [54].

In patients with breast cancer, tumor aggressiveness was reportedly enhanced by IL-17A via induction of angiogenic factors such as chemokines and VEGF. We have previously reported that IL-17A production and VEGF serum levels were also increased in patients with breast, gastrointestinal, and ovarian cancers. Production of IL-12, which induces Th1 cells and maintains cell-mediated immune reaction, and proliferation of lymphocytes (as measured by stimulation index (SI)), a marker of cell-mediated immune function, were both shown to decrease along with disease progression. Moreover, production of IL-17A by Th17 cells and VEGF levels correlated positively with levels of MDSCs, NLR, and C-reactive protein, all of which are good parameters of inflammation, and correlated inversely with IL-12 production and SI ([52], Figure 2). Nutritional parameters, including prealbumin, transferrin, and retinol-binding protein, were also shown to be inversely correlated with IL-17A production and VEGF levels in these patients. Very similar results in patients with various types of cancer have been reported by our laboratory, and these strong correlations among immunosuppression, inflammation, and malnutrition involving MDSCs, IL-17A, and VEGF seemed to be present in advanced malignant diseases [49] [50] [51] [52] [53]. Such findings suggest that the axis of IL-17A, MDSC, and VEGF may be activated further in advanced disease states when nutritional impairment is present. That is, this axis appears closely related to nutritional status, and may play a key role in the development of cancer cachexia (Figure 3). On the one 


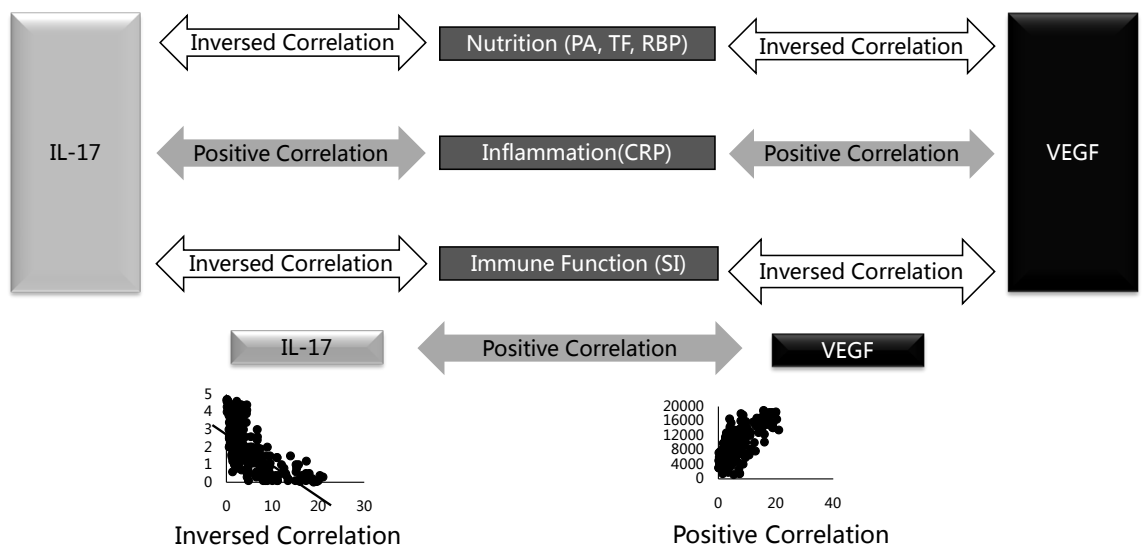

Figure 2. Significant correlations of nutrition, inflammation and immune function with IL-17 and with VEGF in cancer patients. Nutritional condition judged by the levels of PA, TF, RBP is inversely correlated with IL-17 production and with VEGF. Inflammatory condition with the levels of CRP is significantly correlated with IL-17 production by PBMC (peripheral blood lymphocytes) and serum levels of VEGF. Immune function judged by PHA-stimulated proliferation of lymphocytes (SI: stimulation index) is inversely correlated with IL-17 production and VEGF. IL-17 production and VEGF are significantly correlated each other in patients with cancer. Typical inversed correlation and (positive) correlation are graphically shown. PA: prealbumin, TF: transferrin, RBP: retinol binding protein, CRP: C-reactive protein, SI: stimulation index. VEGF: vascular endothelial growth factor.
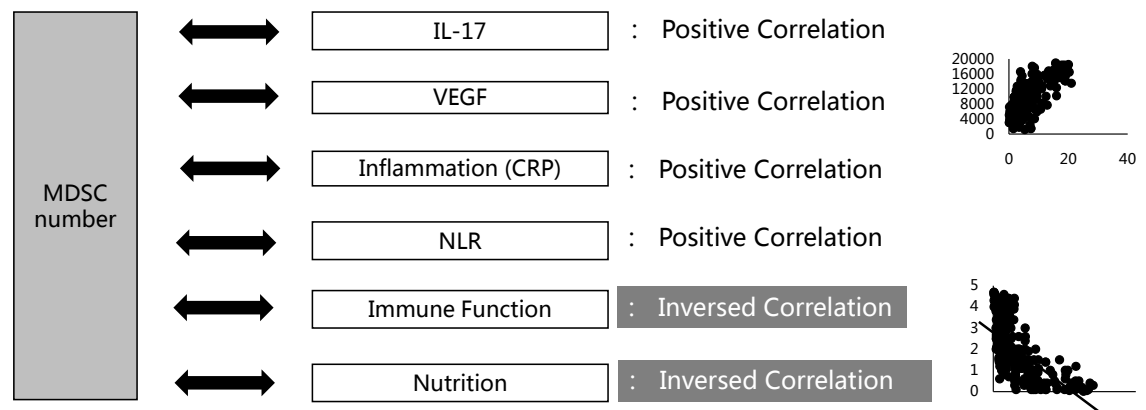

Figure 3. Significant correlations of circulating levels of MDSC with IL-17, VEGF, inflammation, NLR, immune function and nutrition in cancer patients. The circulating levels of MDSC obtained by flow cytometry are significantly correlated with IL-17 production by PBMC (peripheral blood mononuclear cells), serum levels of VEGF, inflammatory condition as the levels of CRP, and neutrophil-to lymphocyte ratios (NLR: one of the inflammatory marker). MDSC levels were also inversely correlated with immune function judged by PHA-stimulated proliferation of lymphocytes (SI: stimulation index), and nutritional condition as the levels of prealbumin, transferrin and retinol binding protein. Typical positive correlation and inversed correlation are graphically shown. MDSC: myeloid-derived suppressor cells, VEGF: vascular endothelial growth factor, CRP: C-reactive protein, NLR: neutrophil to lymphocyte ratio.

hand, Xiang et al. [34] reported that IL-17A has been shown to induce tumor-specific cytotoxic T-lymphocyte activation through the induction of IL-6 and IL-12, and facilitates the maturation of dendritic cells. IL-17 is also necessary for the efficient killing of ovarian CSCs by $\gamma \delta \mathrm{T}$ cells. Lan et al. [55] reported 
that a high density of IL-17-producing cells was associated with improved prognosis of advanced epithelial ovarian cancer. Droeser et al. [56] reported that the combination of myeloperoxidase-positive cell density and IL-17 expression enhanced the indicative value for the response of ovarian carcinoma to chemotherapy, and also offers prognostic value. Taken together, the real clinical picture of IL-17A-driven antitumor activity of the cancer-bearing host has yet to be clearly shown. This activity might depend on the state of disease progression, and needs to be investigated in a larger study population.

\section{Inhibition of IL-17A as a Therapeutic Approach for Cancer}

Therapeutic inhibition of Th17-associated cytokines may be of benefit in oncology. IL-17A, IL-22, and IL-23 may represent target cytokines. For IL-17A, secukinumab and ixekizumab represent two FDA (US food and drug administration)-approved IL-17A inhibitors. However, no clinical trials have been conducted thus far for these agents in cancer settings. Ustekinumab and brodalumab have also been developed as antibodies against IL-17A and have proven to be safe for use [57].

Other than monoclonal antibodies, highly specific and potent inhibitors targeting the Th17-specific transcription factor ROR $\gamma t$ have been identified and are regarded as highly effective [58].

\section{Concluding Remarks}

The pathogenic features of Th17 and IL-17A cells in cancer are still controversial, and Th17 cells appear to promote disease progression, as well as be present in the vicinity of many types of malignant diseases. Th17 differentiation is complex and implies a series of positive and negative regulators that can be therapeutically targeted. MDSC and VEGF are shown to correlate each other and strongly connected to IL-17-driven inflammation, disease progression and malnutrition. Both pre-clinical research and clinical trials are underway to test these concepts. Several strategies utilizing the inhibition of Th17 and IL-17A have arisen, and clinical trials of these complexes are still at an early stage. Increasing our understanding of the interactions between IL-17A and human disorders such as ovarian cancer could lead to new therapeutic strategies in oncology and currently, this area provides a general hope for clinical developments in ovarian cancer.

\section{Conflicts of Interest}

The authors declare no conflicts of interest regarding the publication of this paper.

\section{References}

[1] American Cancer Society (2017) Cancer Facts and Figures. Genes \& Development, 21, 2525-2538. https://doi.org/10.1101/gad.1593107 
[2] Rizzuto, I., Stavraka, C., Chatterjee, J., et al. (2015) Risk of Ovarian Cancer Relapse Score: A Prognostic Algorithm to Predict Relapse Following Treatment for Advanced Ovarian Cancer. International Journal of Gynecologic Cancer, 25, 416-422. https://doi.org/10.1097/IGC.0000000000000361

[3] Hanahan, D. and Weinberg, R.A. (2011) Hallmarks of Cancer: The Next Generation. Cell, 144, 646-674. https://doi.org/10.1016/j.cell.2011.02.013

[4] Bindea, G., Mlecnik, B., Tosolini, M., et al. (2013) Spatiotemporal Dynamics of Intratumoral Immune Cells Reveal the Immune Landscape in Human Cancer. Immunity, 39, 782-795. https://doi.org/10.1016/j.immuni.2013.10.003

[5] Chen, K. and Kolls, J.K. (2017) Interleukin-17A (IL-17A). Gene, 614, 8-14. https://doi.org/10.1016/j.gene.2017.01.016

[6] Fossiez, F., Djossou, O., Chomarat, P., et al. (1996) T Cell Interleukin-17 Induces Stromal Cells to Produce Proinflammatory and Hematopoietic Cytokines. Journal of Experimental Medicine, 183, 2593-2603. https://doi.org/10.1084/jem.183.6.2593

[7] Harrington, L.E., Hatton, R.D., Mangan, P.R., et al. (2005) Interleukin-17-Producing CD4+ Effector T Cells Develop via a Lineage Distinct from the T Helper Type 1 and 2 Lineages. Nature Immunology, 6, 1123-1132. https://doi.org/10.1038/ni1254

[8] Mathur, A.N., Chang, H.C., Zisoulis, D.G., et al. (2017) Stat3 and Stat4 Direct Development of IL-17-Secreting Th Cells. Journal of Immunology, 178, 4901-4907. https://doi.org/10.4049/jimmunol.178.8.4901

[9] Ivanov II McKenzie, B.S., Zhou, L., Tadokoro, C.E., et al. (2006) The Orphan Nuclear Receptor ROR $\gamma$ t Directs the Differentiation Program of Proinflammatory IL-17 ${ }^{+}$T Helper Cells. Cell, 126, 1121-1133. https://doi.org/10.1016/j.cell.2006.07.035

[10] Yao, Z., Fanslow, W.C., Seldin, M.F., et al. (1995) Herpesvirus Saimiri Encodes a New Cytokine, IL-17, Which Binds to a Novel Cytokine Receptor. Immunity, 3, 811-821. https://doi.org/10.1016/1074-7613(95)90070-5

[11] Gaffen, S.L. (2009) Structure and Signaling in the IL-17 Receptor Family. Nature Review of Immunology, 9, 556-567. https://doi.org/10.1038/nri2586

[12] Cua, D.J. and Tato, C.M. (2010) Innate IL-17-Producing Cells: The Sentinels of the Immune System. Nature Review of Immunology, 10, 479-489.

https://doi.org/10.1038/nri2800

[13] Shabgah, A.G., Fattahi, E. and Shahneh, F.Z. (2014) Interleukin-17 in Human Inflammatory Diseases. Advances in Dermatology and Allergology, 31, 256-261. https://doi.org/10.5114/pdia.2014.40954

[14] Rankin, L.C., Girard-Madoux, M.J., Seillet, C., et al. (2016) Complementarity and Redundancy of IL-22-Producing Innate Lymphoid Cells. Nature Immunology, 17, 179-186. https://doi.org/10.1038/ni.3332

[15] Takahashi, N., Vanlaere, I., de Rycke, R., et al. (2008) IL-17 Produced by Paneth Cells Drives TNF-Induced Shock. Journal of Experimental Medicine, 205, 1755-1761. https://doi.org/10.1084/jem.20080588

[16] Kumar, P., Monin, L., Castillo, P., et al. (2016) Intestinal Interleukin-17 Receptor Signaling Mediates Reciprocal Control of the Gut Microbiota and Autoimmune Inflammation. Immunity, 44, 659-671. https://doi.org/10.1016/j.immuni.2016.02.007

[17] Crowe, C.R., Chen, K., Pociask, D.A., et al. (2009) Critical Role of IL-17RA in Immunopathology of Influenza Infection. Journal of Immunology, 183, 5301-5310. https://doi.org/10.4049/jimmunol.0900995

[18] Sears, C.L. and Garett, W.S. (2014) Microbes, Microbiota, and Colon Cancer. Cell 
Host and Microbe, 15, 317-328. https://doi.org/10.1016/j.chom.2014.02.007

[19] Wang, K., Kim, M.K., Di Carlo, G., et al. (2014) Interleukin 17 Receptor Signaling in Transformed Enterocytes Promotes Early Colorectal Tumorigenesis. Immunity, 41, 1052-1063. https://doi.org/10.1016/j.immuni.2014.11.009

[20] Bagheri, N., Azadegan-Dehkordi, F., Shirzad, H., et al. (2015) The Biological Functions of IL-17 in Different Clinical Expressions of Helicobacter pylori-Infection. Microbial Pathogenesis, 81, 33-38. https://doi.org/10.1016/j.micpath.2015.03.010

[21] Atarashi, K., Tanoue, T., Ando, M., et al. (2015) Th17 Cell Induction by Adhesion of Microbes to Intestinal Epithelial Cells. Cell, 163, 367-380. https://doi.org/10.1016/j.cell.2015.08.058

[22] Korn, T., Bettelli, E., Oukka, M. and Kuchroo, V.K. (2009) IL-17 and Th17 Cells. Annual Review of Immunology, 27, 485-517. https://doi.org/10.1146/annurev.immunol.021908.132710

[23] Park, H., Li, Z., Yang, X.O., et al. (2005) A Distinct Lineage of CD4 T Cells Regulates Tissue Inflammation by Producing Interleukin 17. Nature Immunology, 6, 1133-1141. https://doi.org/10.1038/ni1261

[24] Matusevicius, D., Kivisakk, P., He, B., et al. (1999) Interleukin-17 mRNA Expression in Blood and CSF Mononuclear Cells Is Augmented in Multiple Sclerosis. Multiple Sclerosis, 5, 101-104. https://doi.org/10.1177/135245859900500206

[25] Lowes, M.A., Bowcock, A.M. and Krueger, J.G. (2007) Pathogenesis and Therapy of Psoriasis. Nature, 445, 866-873. https://doi.org/10.1038/nature05663

[26] Di Cesare, A., Di Meglio, P. and Nestle, F.O. (2009) The IL-23/Th17 Axis in the Immunopathogenesis of Psoriasis. Journal of Investigative Dermatology, 129, 1339-1350. https://doi.org/10.1038/jid.2009.59

[27] Kirkham, B.W., Lassere, M.N., Edmonds, J.P., et al. (2006) Synovial Membrane Cytokine Expression Is Predictive of Joint Damage Progression in Rheumatoid Arthritis: A Two-Year Prospective Study (the DAMAGE Study Cohort). Arthritis and Rheumatology, 54, 1121-1131. https://doi.org/10.1002/art.21749

[28] Genovese, M.C., Durez, P., Richards, H.B., et al. (2014) One-Year Efficacy and Safety Results of Secukinumab in Patients with Rheumatoid Arthritis: Phase II, Dose-Finding, Double Blind, Randomized, Placebo-Controlled Study. Journal of Rheumatology, 41, 414-421. https://doi.org/10.3899/jrheum.130637

[29] Monteleone, I., Sarra, M., Pallone, F. and Monteleone, G. (2012) Th17-Related Cytokines in Inflammatory Bowel Diseases: Friends or Foes? Current Molecular Medicine, 12, 592-597. https://doi.org/10.2174/156652412800620066

[30] Hueber, W., Sands, B.E., Lewitzky, S., et al. (2012) Secukinumab, a Human Anti-IL-17A Monoclonal Antibody, for Moderate to Severe Crohn's Disease: Unexpected Results of a Randomised, Double-Blind Placebo Controlled Trial. Gut, 61, 1693-1700. https://doi.org/10.1136/gutjnl-2011-301668

[31] Zhao, F., Hoechst, B., Gamrekelashvili, J., et al. (2008) Human CCR4+CCR6+Th17 Cells Suppress Autologous CD8+T Cell Responses. Journal of Immunology, 188, 6055-6062. https://doi.org/10.4049/jimmunol.1102918

[32] Mantovani, A., Allavena, P., Sica, A. and Balkwill, F. (2008) Cancer-Related Inflammation. Nature, 454, 436-444. https://doi.org/10.1038/nature07205

[33] Pasquier, J. and Rafii, A. (2013) Role of the Microenvironment in Ovarian Cancer Stem Cell Maintenance. BioMed Research International, 2013, Article ID: 630782. https://doi.org/10.1155/2013/630782

[34] Xiang, T., Long, H., He, L., et al. (2015) Interleukin-17 Produced by Tumor Micro- 
environment Promotes Self-Renewal of CD133+ Cancer Stem-Like Cells in Ovarian Cancer. Oncogene, 34, 165-176. https://doi.org/10.1038/onc.2013.537

[35] Yamada, Y., Saito, H. and Ikeguchi, M. (2012) Prevalence and Clinical Relevance of Th17 in Patients with Gastric Cancer. Journal of Surgical Research, 178, 685-691. https://doi.org/10.1016/j.jss.2012.07.055

[36] Miyahara, Y., Odunsi, K., Chen, W., et al. (2008) Generation and Regulation of Human CD4+IL-17 Producing T Cells in Ovarian Cancer. Proceedings of the National Academy of Science of USA, 105, 15505-15510.

https://doi.org/10.1073/pnas.0710686105

[37] Maruyama, T., Kono, K., Mizukami, Y., et al. (2010) Distribution of Th17 Cells and FoxP3(+) Regulatory T Cells in Tumor-Infiltrating Lymphocytes, Tumor-Draining Lymph Nodes and Peripheral Blood Lymphocytes in Patients with Gastric Cancer. Cancer Science, 101, 1947-1954. https://doi.org/10.1111/j.1349-7006.2010.01624.x

[38] Tosolini, M., Kirilovsky, A., Mlecnik, B., et al. (2011) Clinical Impact of Different Classes of Infiltrating T Cytotoxic and Helper Cells (Th1, th2, treg, th17) in Patients with Colorectal Cancer. Cancer Research, 71, 1263-1271.

https://doi.org/10.1158/0008-5472.CAN-10-2907

[39] He, S., Fei, M., Wu, Y., et al. (2011) Distribution and Clinical Significance of Th17 Cells in the Tumor Microenvironment and Peripheral Blood of Pancreatic Cancer Patients. International Journal of Molecular Sciences, 12, 7424-7437. https://doi.org/10.3390/ijms12117424

[40] Su, X., Ye, J., Hsueh, E.C., et al. (2010) Tumor Microenvironments Direct the Recruitment and Expansion of Human Th17 Cells. The Journal of Immunology, 184, 1630-1641. https://doi.org/10.4049/jimmunol.0902813

[41] Wang, L., Yi, T., Kortylewski, M., et al. (2009) IL-17 Can Promote Tumor Growth through an IL-6-Stat3 Signaling Pathway. Journal of Experimental Medicine, 206, 1457-1464. https://doi.org/10.1084/jem.20090207

[42] Liu, J., Duan, Y., Cheng, X., et al. (2011) IL-17 Is Associated with Poor Prognosis and Promotes Angiogenesis via Stimulating VEGF Production of Cancer Cells in Colorectal Carcinoma. Biochemical and Biophysical Research Communications, 407, 348-354. https://doi.org/10.1016/j.bbrc.2011.03.021

[43] Talmadge, J.E. and Gabrilovich, D.I. (2013) History of Myeloid-Derived Suppressor Cells. Nature Reviews Cancer, 13, 739-752. https://doi.org/10.1038/nrc3581

[44] Zea, A.H., Rodriguez, P.C., Atkins, M.B., et al. (2005) Arginase-Producing Myeloid Suppressor Cells in Renal Cell Carcinoma Patients. Cancer Research, 65, 3044-3048. https://doi.org/10.1158/0008-5472.CAN-04-4505

[45] Ochoa, A.C., Zea, A.H., Hernandez, C. and Rodriguez, P.C. (2007) Arginase, Prostaglandins, and Myeloid-Derived Suppressor Cells in Renal Cell Carcinoma. Clinical Cancer Research, 13, 721s-726s. https://doi.org/10.1158/1078-0432.CCR-06-2197

[46] Diaz-Montero, C.M., Salem, M.L., Nishimura, M.I., et al. (2009) Increased Circulating Myeloid-Derived Suppressor Cells Correlate with Clinical Cancer Stage, Metastatic Tumor Burden, and Doxorubicin-Cyclophosphamide Chemotherapy. Cancer Immunology and Immunotherapy, 58, 49-59.

https://doi.org/10.1007/s00262-008-0523-4

[47] Gabrilovich, D.I. and Nagaraj, S. (2009) Myeloid-Derived Suppressor Cells as Regulators of the Immune System. Nature Reviews Immunology, 9, 162-174. https://doi.org/10.1038/nri2506

[48] Ostrand-Rosenberg, S. and Sinha, P. (2009) Myeloid-Derived Suppressor Cells: 
Linking Inflammation and Cancer. Journal of Immunology, 182, 4499-4506. https://doi.org/10.4049/jimmunol.0802740

[49] Suzuki, S., Shibata, M., Gonda, K., et al. (2013) Immunosuppression Involving Increased Myeloid-Derived Suppressor Cell Levels, Systemic Inflammation, and Hypoalbuminemia Are Present in Patients with Anaplastic Thyroid Cancer. Molecular and Clinical Oncology, 1, 959-964. https://doi.org/10.3892/mco.2013.170

[50] Yazawa, T., Shibata, M., Gonda, K., et al. (2013) Increased IL-17 Production Correlated with Immunosuppression Involving Myeloid-Derived Suppressor Cells and Nutritional Impairment in Patients with Various Gastrointestinal Cancers. Molecular and Clinical Oncology, 1, 675-679. https://doi.org/10.3892/mco.2013.134

[51] Ohki, S., Shibata, M., Gonda, K., et al. (2012) Circulating Myeloid-Derived Suppressor Cells Are Increased and Correlate to Immune Suppression, Inflammation and Hypoproteinemia in Patients with Cancer. Oncology Reports, 28, 453-458. https://doi.org/10.3892/or.2012.1812

[52] Tachibana, K., Shibata, M., Gonda, K., et al. (2017) IL-17 and VEGF Are Increased and Correlated to Systemic Inflammation, Immune Suppression, and Malnutrition in Patients with Breast Cancer. European Journal of Inflammation, 20, 1-10. https://doi.org/10.1177/1721727X17739514

[53] Minamikawa, K., Shibata, M., Gonda, K., et al. (2017) IL-17 and VEGF Are Significantly Associated with Disease Progression Involving Systemic Inflammation in $\mathrm{Pa}$ tients with Gastric and Colorectal Cancers. Annals Cancer Research and Therapy, 25, 67-76. https://doi.org/10.4993/acrt.25.67

[54] Aotsuka, A., Matsumoto, Y., Arimoto, T., et al. (2019) Interleukin-17 Is Associated with Expression of Programmed Cell Death 1 Ligand 1 in Ovarian Carcinoma. Cancer Science, 110, 3068-3078. https://doi.org/10.1111/cas.14174

[55] Lan, C., Huang, X., Lin, S., et al. (2013) High Density of IL-17 Producing Cells Is Associated with Improved Prognosis for Advanced Epithelial Ovarian Cancer. Cell and Tissue Research, 352, 351-359. https://doi.org/10.1007/s00441-013-1567-0

[56] Droeser, R.A., Mechera, R., Daster, S., et al. (2016) MPO Density in Primary Cancer Biopsies of Ovarian Carcinoma Enhances the Indicative Value of IL-17 for Chemosensitivity. BMC Cancer, 16, 639. https://doi.org/10.1186/s12885-016-2673-7

[57] Rungapiromnan, W., Yiu, Z.Z.N., Warren, R.B., et al. (2017) Impact of Biologic Therapies on Risk of Major Adverse Cardiovascular Events in Patients with Psoriasis: Systematic Review and Meta-Analysis of Randomized Controlled Trials. British Journal of Dermatology, 176, 890-901. https://doi.org/10.1111/bjd.14964

[58] Huh, J.R. and Littman, D.R. (2012) Small Molecule Inhibitors of ROR $\gamma$ t: Targeting Th17 Cells and Other Applications. European Journal of Immunology, 42, 2232-2237. https://doi.org/10.1002/eji.201242740 